\title{
Patent Foramen Ovale Closure-Addressing the Unmet Need for Reducing the Risk of Ischemic Stroke
}

\author{
Hans-Christoph Diener, ${ }^{1}$ Bert Albers, ${ }^{2}$ and Richard W Smalling ${ }^{3}$ \\ 1. Faculty of Medicine, University of Duisburg-Essen, Essen, Germany; 2. Albers Clinical Evidence Consultancy, Winterswijk Woold, The Netherlands; \\ 3. McGovern Medical School at UTHealth, Memorial Hermann Heart and Vascular Institute, Houston, TX, USA
}

C ryptogenic stroke - that is, stroke of an undefined origin, despite thorough investigation—is relatively common, accounting for around one-quarter of cerebral ischemic events. People under 55 years of age are relatively more likely to experience cryptogenic stroke than older individuals. In many patients, the presence of a patent foramen ovale (PFO) may contribute to the risk of cryptogenic stroke; a PFO allows dislodged venous thrombi to pass from the right atrium to the left atrium. This is the basis of paradoxical embolism, and the resulting cerebral artery occlusion may lead to stroke. Treatment to reduce the risk of recurrent cryptogenic stroke uses antithrombotic agents and, increasingly, the interventional option of PFO closure using a catheter-based closure device. Following early clinical trials, the benefit of PFO closure was relatively uncertain. Emerging evidence from longer-term studies published in recent years, however, has presented a clear case for the benefits of PFO closure in selected patients under the age of 60 years in terms of reducing risk of recurrent stroke. Treatment guidelines are being updated to reflect these findings.

\section{Keywords}

PFO closure, patent foramen ovale, cryptogenic stroke

Disclosures: Hans-Christoph Diener has received honoraria for participation in clinical trials, contribution to advisory boards or oral presentations from: Abbott, Bayer Vital, Bristol Myers Squibb, Boehringer Ingelheim, Daiichi-Sankyo, Medtronic, Pfizer, Portola, Sanofi-Aventis, and WebMD Global. Financial support for research projects has been provided by Boehringer Ingelheim. Hans-Christoph Diener chairs the Treatment Guidelines Committee of the German Society of Neurology and contributed to the European Heart Rhythm Association and European Society of Cardiology guidelines for the treatment of atrial fibrillation. Bert Albers has received honoraria from Abbott Vascular for medical writing support. Richard W Smalling is a consultant for, and receives grant support from, Abbott Vascular

Acknowledgments: Medical writing support, including preparation of the drafts under the guidance of the authors, was provided by Colin Griffin of Touch Medical Communications, and funded by Abbott.

Review Process: Double-blind peer review.

Compliance with Ethics: This article involves a review of the literature and did not involve any studies with human or animal subjects performed by any of the authors.

Authorship: The named authors meet the International Committee of Medical Journal Editors (ICMJE) criteria for authorship of this manuscript, take responsibility for the integrity of the work as a whole, and have given final approval for the version to be published.

Access: This article is freely accessible at touchNEUROLOGY.com (C) Touch Medical Media 2020.

Received: February 27, 2020

Accepted: June 1, 2020

Published Online: July 10, 2020

Citation: US Neurology. 2020;16(1):54-60

Corresponding Author: Hans-Christoph Diener, Faculty of Medicine, University of Duisburg-Essen, 45147 Essen, Germany. E: Hans.Diener@uk-essen.de

Support: The publication of this article was supported by Abbott, who was given the opportunity to review the article for scientific accuracy before

submission. Any resulting changes were made at the authors' discretion.
Globally, a high proportion (around 25\%) of transient ischemic attacks (TIAS) and ischemic strokes are cryptogenic., The Trial of ORG 10172 in Acute Stroke Treatment (TOAST) classification defines a cryptogenic stroke as a brain infarction that is not caused by definite cardioembolism, large artery atherosclerosis, or small artery disease, despite extensive investigations. ${ }^{3}$ The "ASCOD" classification defines strokes by subtype causes: (A) atherosclerosis, (S) small vessel disease, (C) cardiac disease, (O) other cause, or (D) dissection; a cryptogenic stroke is an event that is of completely unknown cause in that it does not conform to any of these subtypes. ${ }^{4-6}$ The lack of consistent criteria for cryptogenic stroke, and the fact that many TIAs are inappropriately described as cryptogenic stroke due to incomplete work-up, makes accurately defining cryptogenic stroke incidence challenging. ${ }^{5}$ Therefore, the concept of embolic stroke of undetermined source (ESUS) was introduced, which provides an operational definition and requires predefined diagnostic procedures. ${ }^{2}$ However, across Europe, it is estimated that as many as one-third of TIAs and strokes are cryptogenic, accounting for around 400,000 events annually. ${ }^{1}$ Similarly, in the USA, cryptogenic stroke may account for up to $30 \%$ of approximately 690,000 overall ischemic stroke events per year. ${ }^{7}$ In many cases, cryptogenic stroke is treated with antiplatelet therapy where there is an absence of concomitant atrial fibrillation.?

In younger patients (those $<60$ years), the incidence of cryptogenic stroke is relatively greater than in older patients, ${ }^{5}$ occurring in approximately half of younger stroke cases in a recent population-based study. ${ }^{1}$ The high incidence of cryptogenic stroke, combined with the uncertain cause of stroke, complicates secondary prevention strategies that focus on reducing the risk of future strokes. ${ }^{1}$ Cryptogenic strokes and ESUS are considered to have several potential underlying causes of thromboembolism, including occult arterial sources of thromboembolism, asymptomatic paroxysmal atrial fibrillation, minor-risk cardiac structural abnormalities or paradoxical embolism of venous emboli through a patent foramen ovale (PFO). ${ }^{1,25,8}$ 
Table 1: The association of patent foramen ovale with cryptogenic stroke has been repeatedly confirmed

\begin{tabular}{|c|c|c|c|c|c|}
\hline Study & Patients, N & Age, years & Cryptogenic, \% (n/N) & Control, \% (n/N) & $p$ value \\
\hline \multicolumn{6}{|l|}{ Younger patients } \\
\hline Lechat et al. & 26 & $<55$ & $54(14 / 26)$ & $10(10 / 100)$ & $<0.001$ \\
\hline Webster et al. & 34 & $<40$ & $56(19 / 34)$ & $15(6 / 40)$ & $<0.001$ \\
\hline Cabanes et al. & 64 & $<55$ & $56(36 / 64)$ & $18(9 / 50)$ & $<0.001$ \\
\hline De Belder et al.* & 39 & $<55$ & $13(5 / 39)$ & $3(1 / 39)$ & - \\
\hline Di Tullio et al. & 21 & $<55$ & $47(10 / 21)$ & $4(1 / 24)$ & $<0.001$ \\
\hline Hausmann et al. & 18 & $<40$ & $50(9 / 18)$ & $11(2 / 18)$ & $<0.050$ \\
\hline Total & - & - & $46(93 / 202)$ & $11(29 / 271)$ & - \\
\hline \multicolumn{6}{|l|}{ Older patients } \\
\hline De Belder et al.* & 64 & $>55$ & $20(13 / 64)$ & $5(3 / 56)$ & $<0.001$ \\
\hline Di Tullio et al. & 24 & $>55$ & $38(9 / 24)$ & $8(6 / 77)$ & $<0.001$ \\
\hline Hausmann et al. & 20 & $>40$ & $15(3 / 20)$ & $23(23 / 98)$ & NS \\
\hline Jones et al. & 57 & $>50$ & $18(10 / 57)$ & $16(29 / 183)$ & NS \\
\hline Total & - & - & $21(35 / 165)$ & $15(61 / 414)$ & - \\
\hline
\end{tabular}

*Includes different stroke subtypes.

NS = not significant.

Reproduced with permission from Homma et al. $2005 .^{9}$

PFO is the most common congenital cardiac abnormality, and is present in approximately $25 \%$ of the adult population. ${ }^{9}$ The foramen ovale is an obligatory channel that allows placental oxygenated blood to reach the arterial circulation of the fetus, which closes spontaneously, postnatally, in around $75 \%$ of the population. ${ }^{9}$ A PFO occurs in adults who have incomplete postnatal fusion of the septum primum and secundum, ${ }^{10,11}$ and presents a pathway for paradoxical embolization. ${ }^{9}$ The prevalence of PFO among patients with cryptogenic stroke is relatively high, with a median prevalence of approximately $40-60 \% .{ }^{12}$ Compared with older patients, PFOs are more frequent in younger cryptogenic stroke cases; ${ }^{5,11,13,14}$ as shown in a systematic review of 23 case-controlled studies, which found that younger ( $<55$ years) patients with cryptogenic stroke had over two-fold higher odds of presenting with a PFO than older patients..$^{15}$

\section{Patent foramen ovale as a risk factor for cryptogenic stroke}

The association of PFO with cryptogenic stroke has been repeatedly confirmed, with particularly robust evidence apparent in the younger population; however, PFO in older stroke patients is also important (Table 1). 9,13,16 It may be considered that PFO is associated with cryptogenic stroke independent of age, but that the effect is stronger in patients $\leq 60$ years of age. ${ }^{9}$

The presence of PFO allows intracardiac shunting when right atrial pressure exceeds left atrial pressure (e.g., at the end of a Valsalva maneuver), which is associated with up to $95 \%$ of right-to-left shunts. ${ }^{16}$ such increased right atrial pressure can arise from everyday activities, such as exercise, coughing, or defecating. The right-to-left shunt associated with PFO can allow migration of venous thrombi from the right to the left atrium (paradoxical embolism), thereby bypassing filtration in the pulmonary vasculature. This increases the risk of cerebral artery blockage and subsequent stroke. ${ }^{11}$ Venous thrombosis in the legs or pelvis, particularly in patients with deep vein thrombosis (DVT) is a common source of emboli. ${ }^{17}$ In patients, especially those who are older, with larger PFO and concomitant DVT, the risk of stroke is increased where a dislodged venous thrombus can shunt from the venous system to the cerebral arteries. ${ }^{18}$ Significant risk is posed by paradoxical embolism with thrombi of any size. ${ }^{18}$ In studies, DVT has been detected in 7.6-9.5\% of patients with PFO and a stroke event. ${ }^{5}$ Importantly, it is estimated that up to $80 \%$ of DVT is asymptomatic, which highlights the need for thorough investigation in patients with PFO and cryptogenic stroke, who are at elevated risk of paradoxical embolism. ${ }^{5}$ An additional practical consideration is that DVT may develop in patients who are bed-ridden, post-stroke. ${ }^{5}$ The annual risk attributed to paradoxical embolism in people with PFO has been estimated at 28 per 100,000 people, which is a sizeable proportion of the overall first ischemic stroke rate of 139 per 100,000 people per year in the European population. ${ }^{16}$

Atrial septal aneurysm (ASA), where the atrial septum primum is abnormally redundant and bulges into the right or left atrium during cardiac contraction, is also associated with PFO in $20-40 \%$ of patients. ${ }^{19}$ With each heartbeat, the ASA may open the PFO, potentially increasing the likelihood of paradoxical embolism, and thus the presence of an ASA correlates with increased risk of an initial stroke from paradoxical embolism. ${ }^{20}$

Historically, there has been debate over the role of PFO in recurrent stroke, with several studies observing no significant association. 21,22 The presence of both PFO and ASA was significantly associated with increased risk of recurrent stroke, ${ }^{23}$ because an ASA may interact with the PFO and potentiate stroke risk. ${ }^{11}$ Case-control studies have suggested that PFO prevalence may be four-fold higher in younger patients with stroke and two-fold higher in older patients, compared with similar-aged control participants. ${ }^{24}$ Moreover, recent analyses have demonstrated significant benefit in the reduction of recurrent cryptogenic stroke or composite measures of stroke and TIA through the management of PFO, via PFO closure. ${ }^{20,25-27}$ This emphasizes the need for effective early detection of PFO and identification of patients who have experienced cryptogenic stroke for whom PFO closure may be beneficial in reducing risk of future stroke. 


\section{Diagnosis in patients at risk of recurrent cryptogenic stroke with patent foramen ovale}

since individuals who experience cryptogenic stroke are generally relatively young, they are less likely to have classic risk factors for ischemic stroke such as hypertension, hyperlipidemia, and diabetes. ${ }^{1,7}$ PFO is a relatively common finding, so a thorough multi-disciplinary evaluation is important to establish it as the most plausible cause of a stroke. This is of particular importance because in some patients, PFO may be incidental, ${ }^{15}$ and the selected treatment strategy must address the underlying cause of a stroke to be effective.

Complete patient work-up to identify PFO as a cause of cryptogenic stroke or a predictive factor for further stroke events would include: cardiac evaluation (including early rhythm monitoring); 72-hour Holter monitoring and transthoracic or transesophageal echocardiography (to exclude atrial fibrillation and other high-risk cardiac sources of embolism); neurologic imaging, ideally magnetic resonance imaging (MRI; to confirm that the stroke has an embolic presentation); and cervical and cerebral arterial imaging (to confirm that there is no clinically relevant stenosis). ${ }^{57,71,28}$

Transthoracic echocardiogram (TTE) with administration of agitated saline bubbles is often the preferred tool for initial detection of PFO. The "bubble study" observes the timing of bubbles appearing into the left-sided cardiac chambers and is important to differentiate intracardiac from transpulmonary shunts. TTE is best performed with increased pressure in the right atrium, for example, during the release phase of the Valsalva maneuver or with coughing, sniffing, or with the manual application of external abdominal pressure. Transesophageal echocardiogram (TEE) is used to examine the anatomical characteristics of the PFO, including tunnel length, margins, rims, and surrounding structures. TEE is currently considered the "gold standard" in PFO characterization. ${ }^{29}$ The greater anatomical detail provided by TEE can help diagnose multiple defects and may increase the chances of successful PFO closure.

Recent practical guidelines suggest a workflow for assessing risk of recurrent stroke due to $\mathrm{PFO} .^{30}$ This advises that appropriate imaging should be performed, with a recommendation to use TEE with bubble contrast in younger adults with unexplained cerebrovascular events. This is because TEE has higher sensitivity for PFO detection compared with transthoracic imaging. Work-up of the cryptogenic stroke profile should consider atrial septal defect, cardiac tumors, bacterial or non-bacterial valve vegetations, and atrial thrombi in addition to PFO. Detection of PFO should be performed by observing microbubbles entering the right atrium and, in the presence of a PFO, these will pass into the left atrium within a few heartbeats. The sensitivity of detection of right-to-left shunts is increased with cough or Valsalva maneuver during the bubble contrast injection. The guidance notes that, although usual shunting is predominantly left-to-right, there can be some right-to-left shunting as the relative pressures in the two chambers change during the cardiac cycle and with respiration. Detection of an ASA should be considered as an elevating risk factor for stroke in patients with a PFO. ${ }^{30}$

A recent study has shown that the Risk of Paradoxical Embolism (ROPE) score may be used as an independent predictor of recurrent cryptogenic stroke and as a tool for assessing the likely benefit of PFO closure. ${ }^{31}$ The RoPE score is predictive of the likelihood of stroke recurrence after PFO closure, and can help quantify whether PFO may be causative or incidental to the stroke. ${ }^{31}$ However, in practice, physicians may find the RoPE score challenging to use in their patients, and consequently, studies have shown that patients stratified as most likely to experience PFO-related cryptogenic stroke have disproportionately low predicted recurrence rates. Physicians must also consider multiple other factors that increase the likelihood that a stroke is PFO-mediated, including obesity, index stroke severity, a history of DVT or pulmonary embolism, hypercoagulable states, prolonged travel or forced immobility, migraine, or performance of a Valsalva maneuver prior to the stroke event. ${ }^{32}$ These findings reflect the challenges faced by physicians in diagnosing PFO risk and selecting treatment. ${ }^{32}$

\section{Treatment options in the management of recurrent stroke risk}

Clinical decision-making needs to focus on effective early treatment with the goal of preventing future stroke events. This is of particular importance in the younger population to help limit the burden of repeated strokes throughout a patient's subsequent lifetime. Treatment for the management of cryptogenic stroke falls into two major categories: antithrombotic medical therapy and interventional procedures plus medium- to long-term antithrombotics. For antithrombotic treatment, both anticoagulants and antiplatelets are typically used in patients with stroke.,33 ${ }^{3,3}$ significant difference in efficacy has been found between antiplatelets and anticoagulants in ischemic event recurrence, death rates or side-effects between patients with both cryptogenic stroke and PFO. 5,34,35 Since antiplatelets have a lower bleeding risk than vitamin-K antagonists, they may be preferred in practice. ${ }^{34}$ Anticoagulation with dabigatran has demonstrated similar bleeding risk to aspirin, as has rivaroxaban in the subset of patients with $\mathrm{PFO}^{36,37}$ As a result of the lack of differentiating findings, there is little clear guidance on the selection of one of these agents over the other. ${ }^{5,33}$ As a consequence, treatment initiation should reflect the relative risks and benefits of therapy, as discussed with the patient, and in a multidisciplinary setting.

Interventional treatment seeks to close the PFO. Percutaneous PFO closure is performed using a catheter to implant a device to seal the PFO; there are differences in device design that affect ease of delivery, efficacy in achieving complete closure, and adverse effects. ${ }^{11}$ There is an increasing body of evidence that supports the emergence of PFO closure as a key treatment option for reducing risk of recurrent cryptogenic stroke in selected patients, particularly those $<60$ years of age, with PFO. ${ }^{11,20}$ For certain patients with PFO at risk of recurrent stroke, PFO closure in combination with long-term antithrombotic medication may offer significant benefit in reduction of stroke recurrence risk. ${ }^{30,38}$

\section{Emerging evidence base for the benefits of patent foramen ovale closure}

The evidence base drawn from randomized controlled trials of PFO closure has evolved over the past decade. Three trials that published findings in 2012-2013 found little evidence for superiority of PFO closure over antithrombotic therapy.

The Closure or Medical Therapy for Cryptogenic Stroke with Patent Foramen Ovale (CLOSURE) trial was conducted in 909 patients (aged 18-60 years). This study reported a non-significant $22 \%$ reduction in the relative risk (RR) of the primary endpoint (frequency of strokes or TIA in the 2-year follow-up period, all-cause mortality in the first 30 days, or death due to neurological causes between day 31 and 2 years) versus medical treatment alone 
Table 2: Trends for reduction in incidence of stroke, transient ischemic attack, and death were seen in all patent foramen ovale-closure trials, compared with medical treatment alone

\begin{tabular}{|c|c|c|c|c|c|c|c|}
\hline Parameters & & CLOSURE & $\mathrm{PC}$ & RESPECT & REDUCE & CLOSE & DEFENSE-PFO \\
\hline Stroke (\%) & $M$ & 3.1 & 2.4 & 5.8 & 5.4 & 6.0 & 10.5 \\
\hline Stroke (\%) & C & 2.9 & 0.5 & 3.6 & 1.4 & 0 & 0 \\
\hline TIA (\%) & M & 4.1 & 3.3 & 4.8 & - & - & 2.0 \\
\hline TIA (\%) & C & 3.1 & 2.5 & 3.4 & - & - & 0 \\
\hline Death (\%) & M & 0 & 0 & 2.2 & 0 & 0 & 0 \\
\hline Death (\%) & C & 0 & 1.0 & 1.4 & 0.5 & 0 & 0 \\
\hline
\end{tabular}

$C=$ PFO closure; $M=$ medical treatment; PFO = patent foramen ovale; TIA = transient ischemic attack.

Reproduced with permission from Diener et al. 2018.38

(hazard ratio [HR] 0.78; 95\% confidence interval [Cl] 0.45-1.35; $\mathrm{p}=0.37$ ). ${ }^{39}$ In addition, the Percutaneous Closure of Patent Foramen Ovale in Cryptogenic Embolism (PC) trial was conducted in 414 patients (18-60 years of age) and found a non-significant 37\% RR reduction in the composite endpoint of death, non-fatal stroke, TIA, or peripheral embolism, over 4 years versus medical treatment alone (HR 0.63; 95\% $\mathrm{Cl} 0.24-1.62 ; \mathrm{p}=0.34) .{ }^{40}$

These early trials had relatively long initialization periods and relatively short follow-up intervals (45 days to 4 years), post-randomization. Although there were numerical benefits to PFO closure in these studies, ${ }^{38}$ the lack of significant effect led to widespread debate into the value of PFO closure over medical therapy alone. Recent trials, discussed below, have been conducted over longer observation periods (2-6 years) with larger patient populations and using more precise selection of patients, and have contributed more consistent findings to the evidence base for using PFO closure to reduce risk of new or recurrent strokes.

The Closure of Patent Foramen Ovale versus Medical Therapy after Cryptogenic Stroke (RESPECT) trial included 980 patients (18-60 years of age). A significant $63 \%$ RR reduction in a composite endpoint of stroke during the 2-year follow-up period or early all-cause death versus medical treatment alone in the per-protocol analysis (HR 0.37; 95\% Cl 0.14-0.96; $\mathrm{p}=0.03$ ) was observed. However, this improvement was not significant in the intention-to-treat cohort, with nine endpoint events occurring in the PFO closure cohort and 16 in the medical treatment cohort (HR 0.49; 95\% $\mathrm{Cl} 0.22-1.11 ; p=0.08) .{ }^{41}$ Long-term results have now been provided for the RESPECT study; data are available for 980 patients who were observed for a median of 6 years. There was a significant reduction in stroke recurrence in the PFO closure cohort, compared with medical treatment alone (HR $0.55 ; 95 \% \mathrm{Cl} 0.31-0.99 ; \mathrm{p}=0.046)$ and a significant reduction in recurrence of stroke of unknown origin ( $\mathrm{HR} 0.38 ; 95 \% \mathrm{Cl} 0.18-0.79 ; \mathrm{p}=0.007$ ). ${ }^{42.41}$ Over the longer follow-up period, the signal for the probability of freedom from stroke events starts to separate after around 2 years and continues to demonstrate benefit over a 10 -year period. ${ }^{42}$

The Patent Foramen Ovale Closure or Anticoagulants versus Antiplatelet Therapy to Prevent Stroke Recurrence (CLOSE) trial studied 663 patients (16-60 years of age) with an ASA or a large shunt. This population would therefore be at high risk of a paradoxical embolism being the causative mechanism of stroke. Patients were randomized across three treatment groups:

- Randomization group 1: PFO closure plus long-term antiplatelet therapy (PFO closure group), antiplatelet therapy alone, or oral anticoagulation;
- Randomization groups 2 and 3: patients with contraindications to anticoagulants or to PFO closure were randomly assigned to the alternative non-contraindicated treatment, or to antiplatelet therapy.

The CLOSE study demonstrated a significant 97\% RR reduction for fatal and non-fatal stroke in the PFO closure cohort compared with antiplatelet therapy alone over 5.3 years ( $\mathrm{HR} \mathrm{0.03;} \mathrm{95 \%} \mathrm{Cl} \mathrm{0.00-0.26;} \mathrm{p}<0.001$ ). The study reported no stroke events among the patients undergoing PFO closure throughout the follow-up period. There was no significant difference in the primary endpoint outcome between anticoagulant therapy alone or antiplatelet therapy alone. ${ }^{43}$

The REDUCE trial was conducted in 664 patients (18-59 years of age) and observed a significant $77 \%$ RR reduction in recurrent ischemia or incidence of new stokes over 3.2 years of follow-up, with PFO closure plus antiplatelet therapy versus antiplatelet treatment alone (HR 0.23; 95\% Cl 0.09-0.62; $\mathrm{p}=0.002){ }^{44}$ The DEFENSE-PFO study followed 120 patients over 2 years of observation and found a significant reduction ( $p=0.013)$ in the composite of stroke, vascular death, and major bleeding, versus medical treatment alone, recording no events in the PFO closure cohort. Enrollment and progression of this study was terminated early on account of the emerging trial data from CLOSE and REDUCE, which had clearly demonstrated benefits of PFO closure over medical management alone in the relative reduction of risk of stroke. ${ }^{45}$ Overall, trends for reduction in incidence of stroke, TIA, and death were seen in all PFO-closure trials, compared with medical treatment alone (Table 2). It has been observed that CLOSE, REDUCE and the long-term RESPECT datasets showed particularly notable benefits in patients with large shunt volumes (and/or ASA, in RESPECT and CLOSE). ${ }^{38}$

Recent meta-analyses have demonstrated clinically relevant benefit with PFO closure in the reduction of recurrent cryptogenic stroke versus medical therapy alone (Figure 1).11,20,46 A meta-analysis reported that, compared with medical intervention alone, PFO closure was associated with a $57 \%$ reduction in risk of recurrent stroke (RR of recurrent stroke 0.43; 95\% Cl 0.21-0.90; $\mathrm{p}=0.024) .{ }^{25} \mathrm{~A}$ recent analysis concluded that, compared with medical antiplatelet therapy alone, PFO closure plus long-term medical antiplatelet therapy provided a significant $81 \%$ RR reduction in recurrent stroke. ${ }^{11}$ Collectively, the PFO closure trials show effective sealing of PFO, with minimal residual, if any, shunt volumes being detected post-intervention. ${ }^{11}$ Considering all PFO closure study findings, it has been suggested that in routine practice, individuals most likely to benefit from PFO closure are younger ( $<60$ years old), with a RoPE score $\geq 7$ (no other identifiable stroke risk factor), and with neurologic imaging strongly 
Figure 1: Meta-analysis of benefits in recurrent stroke following patent foramen ovale closure

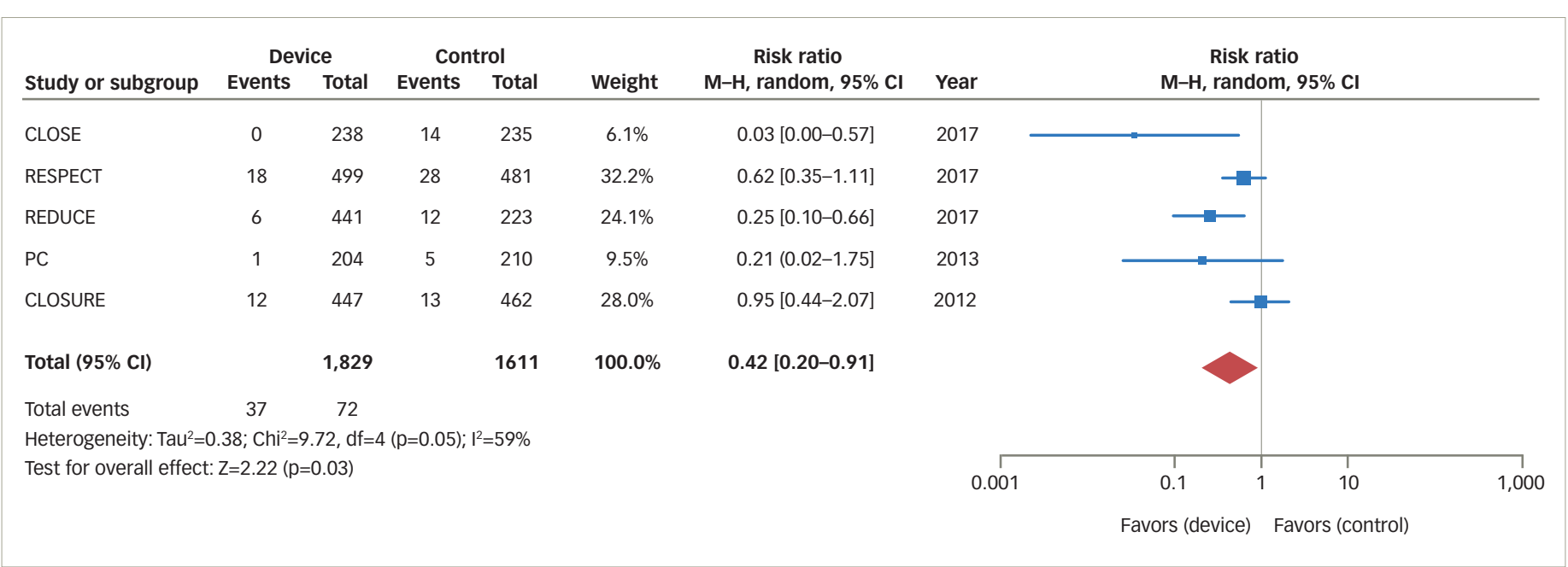

$\mathrm{Cl}=$ confidence interval; $\mathrm{M}-\mathrm{H}=$ Mantel-Haenszel.

Reproduced with permission from Mojadidi et al. 2018. ${ }^{20}$

in favor of a cardioembolic event. ${ }^{27}$ Patients with large shunt volumes are particularly likely to benefit from PFO closure. ${ }^{38}$ Recurrent strokes following PFO closure are typically non-cryptogenic and are associated with atrial fibrillation or conventional vascular risk factors for stroke. ${ }^{27}$

No difference in incidence of bleeding events has been reported in analysis of PFO closure versus medical therapy. ${ }^{12}$ Atrial fibrillation is the most notable adverse event following PFO closure, compared with medical therapy alone. ${ }^{11,12,27,46,47}$ In practical terms, this highlights the need for personalization of treatment selection; physicians must determine the relative benefits of PFO closure versus the potential introduction of a stroke risk factor.

\section{Evolving guidelines for patent foramen ovale closure in the management of cryptogenic stroke risk}

Practical guidelines are evolving to reflect the broadening evidence base for the use of PFO closure in certain patients at relatively high risk of recurrent cryptogenic stroke. The 2018 Canadian Stroke Best Practice Recommendations for the secondary prevention of stroke state that: "There is now sufficient evidence to recommend PFO closure for very carefully selected patients aged 60 years or younger with an unexplained embolic stroke event who are found to have a PFO and who do not require chronic anticoagulant therapy for another reason." ${ }^{48}$ These guidelines recommend antiplatelet therapy without PFO closure for patients $>60$ years of age with a history of stroke, and advocate the same approach in patients for whom the PFO is likely to be incidental. ${ }^{48}$ A 2019 German guideline consensus stated that: "Interventional PFO closure should be performed in patients aged 16 to 60 years (after neurological and cardiological diagnostic work-up) with a history of cryptogenic ischemic stroke and PFO with moderate or extensive right-to-left shunt." ${ }^{\prime 3}$ Where patients are not suited to PFO closure, these recommendations find no superiority with either anticoagulant or antiplatelet therapy. ${ }^{38} \mathrm{~A}$ recent European position paper summarizing the consensus views of eight societies stated that: "The position of our societies is to perform percutaneous closure of a PFO in carefully selected patients aged from 18 to 65 years with a confirmed cryptogenic stroke, TIA, or systemic embolism and an estimated high probability of a causal role of the PFO as assessed by clinical, anatomical and imaging features." ${ }^{47}$ The American Heart Association/American Stroke Association guidelines note that PFO closure may be of benefit to patients with cryptogenic stroke, a PFO, and a history of DVT. ${ }^{33}$

This collection of guidance is broadly echoed in the 2018 British Medical Journal Rapid Recommendations statement on clinical practice guidelines for selecting therapy for patients $<60$ years old with PFO who have had a cryptogenic ischemic stroke, when extensive work-up for other etiologies of stroke is negative. ${ }^{30}$ The current guidance (Figure 2) advocates that for patients who are suited to all treatment options, there is a "weak recommendation for PFO closure plus long-term antiplatelet therapy, rather than anticoagulant therapy." For patients in whom anticoagulation is contraindicated or declined, there is a "strong recommendation for PFO closure plus long-term antiplatelet therapy versus antiplatelet therapy alone." For patients in whom PFO closure is contraindicated or declined, there is a "weak recommendation for anticoagulant therapy rather than antiplatelet therapy. " ${ }^{30}$

The authors of the British Medical Journal Rapid Recommendations statement reiterate the need for personalized treatment. ${ }^{30}$ This is emphasized in findings from the RESPECT trial population, in which there was a higher incidence of DVT and venous thromboembolism (VTE) compared with the general population. This suggests the need for prioritizing anticoagulation therapy over antiplatelet therapy, and may challenge the recommendations that are given in local guidelines. It is considered that patients who show evidence of VTE should receive optimized anticoagulation therapy; ${ }^{49}$ this may be even more important in patients with a PFO. However, there remains a need for more understanding of the safety and efficacy of anticoagulation compared with vitamin $\mathrm{K}$ agonists in patients with PFO; to date, evidence is limited to subgroups of the RE-SPECT ESUS and NAVIGATE ESUS studies. ${ }^{36,37}$

Recent commentary has suggested that guidelines should re-evaluate their management recommendations for patients with PFO-mediated strokes, and noted that cryptogenic stroke, ASCOD and ESUS criteria may underestimate the role of PFO in strokes in real-world practice..$^{50,51}$ 
Figure 2: Clinical practice recommendations for treatment selection in patients with patent foramen ovale and risk of recurrent cryptogenic stroke
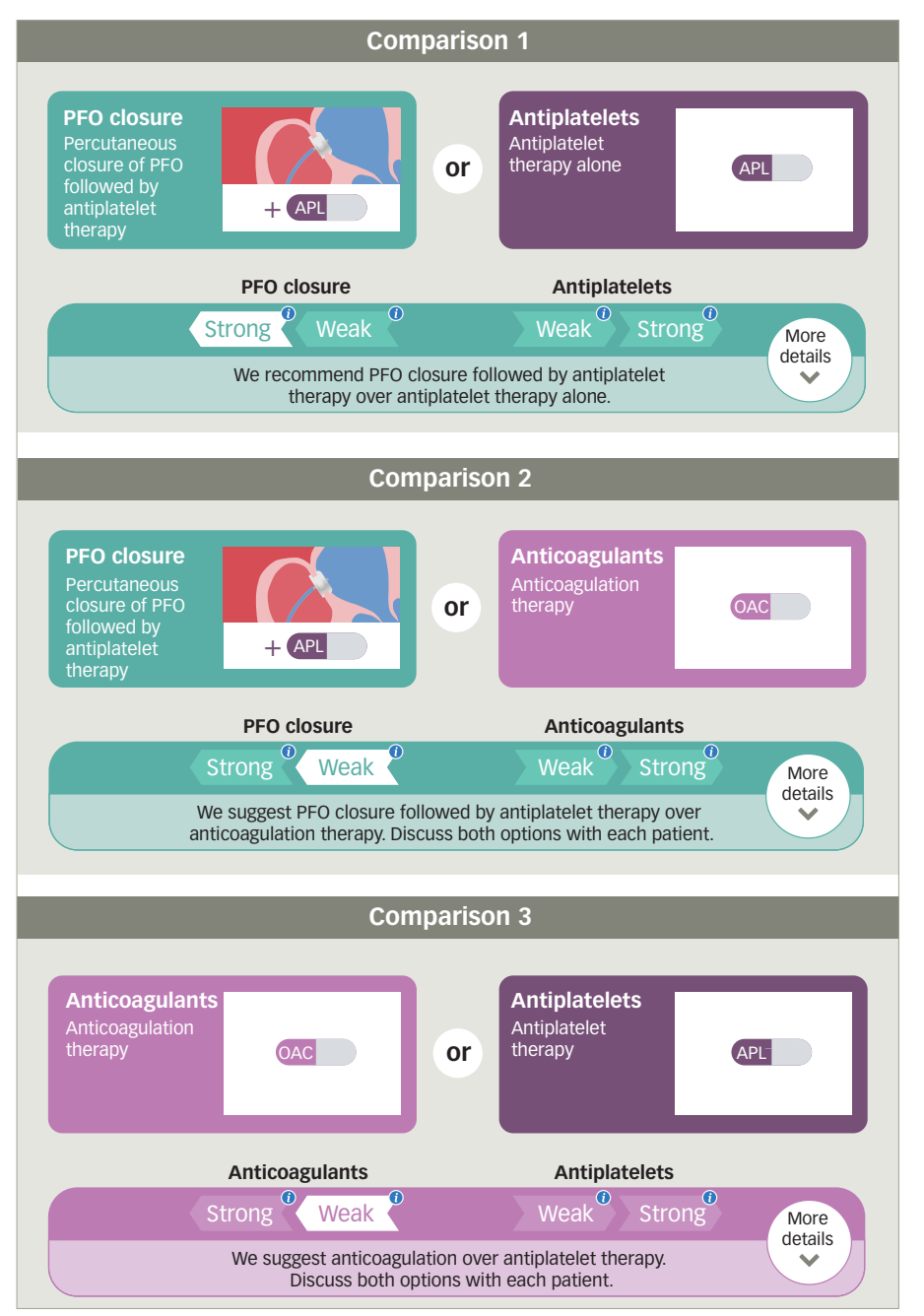

$A P L=$ antiplatelet; $O A C=$ oral anticoagulant; $P F O=$ patent foramen ovale. Reproduced with permission from Kuijpers et al. 2018. ${ }^{30}$

Physicians may consider that PFO is always a potential culprit for stroke, even where other traditional risk factors exist.

\section{Ongoing challenges in the selection of patent foramen ovale closure in patients with cryptogenic stroke}

In routine practice, there remain a number of unresolved issues concerning cryptogenic stroke treatment that require individualized consideration, and, in due course, evidence-based guidance. The selection of medical therapy as an adjunct to PFO closure is contested, with limited data showing an advantage for either antiplatelet or anticoagulant treatment in terms of trial endpoints. ${ }^{14}$ While anticoagulation has benefits in the management of patients with VTE, there are associated risks in terms of increased bleeding. ${ }^{14}$ Moreover, the selection of a non-vitamin $\mathrm{K}$ antagonist oral anticoagulant instead of warfarin has been shown to reduce the risk of stroke in patients with atrial fibrillation, without increased risk of bleeding. This is of particular importance to patients who may develop atrial fibrillation as a consequence of PFO closure.
In patients with atrial fibrillation, apixaban had a comparable bleeding risk to aspirin, ${ }^{52}$ and in the RE-SPECT ESUS trial, dabigatran had a similar bleeding risk to aspirin. ${ }^{36}$

Atrial fibrillation can occur during or acutely after PFO closure, and is related to irritation caused by device implantation. The risk of atrial fibrillation may be slightly elevated during the first 6 weeks after closure, but is considered transitory and the condition generally resolves with or without medical treatments such as beta blockers. In the RESPECT trial, after the periprocedural period, there was no difference in the incidence of atrial fibrillation between the device and the medical treatment arms. ${ }^{41}$ As the PFO population ages, post-closure, it is likely that the incidence of new atrial fibrillation will be no different than that of a similarly aged population, which has not undergone closure. Other complications following implantation, occurring in less than $1 \%$ of cases, may include infection, erosion into the pericardium or aorta at the rim of the device, or formation of a new atrial septal defect caused by tearing of the septum primum by the rim of the device. ${ }^{53}$ Palpitations are not uncommon immediately following implantation, and usually subside spontaneously. ${ }^{53}$ A study of long-term serious complications, defined as: atrial fibrillation or flutter, cardiac tamponade, pneumothorax, hemothorax, a vascular access complication, or death, found a cumulative incidence rate of $16.3 \%$ over the 5 years following implantation, with a mortality rate of $3.4 \% .^{54}$

The role of PFO closure in older patients or patients with a history of stroke of unknown origin is also less well defined; the pivotal randomized controlled trials enrolled only patients $<60$ years of age and generally only included cryptogenic stroke, excluding lacunar strokes. ${ }^{38-45}$ The paucity of data in older patients, or those without clearly defined cryptogenic stroke, makes generalization of study data difficult, hence current guidance must recommend PFO closure in younger patients only, where the PFO is not likely to be incidental. ${ }^{14}$ That said, in individual patients over the age of 60 who have experienced several cryptogenic strokes, particularly when vitamin $\mathrm{K}$ antagonists have been discontinued for surgical procedures, an exception to the previous guidance should be considered. A further unresolved issue is the role of the presence of atherosclerotic plaques in the aortic arch or carotid arteries in patients with PFO.

In future trial design, it may be beneficial for patient selection and inclusion to be based on the ESUS criteria rather than cryptogenic stroke. Diagnosis of ESUS requires brain imaging and exclusion of significant stenosis or occlusion of both extra- and intracranial arteries. These criteria may also help inform patient selection for PFO closure in routine practice. ${ }^{14}$

\section{A multidisciplinary approach to cryptogenic stroke management}

cooperation between neurologists and cardiologists as part of a multidisciplinary team approach to managing risk of recurrent cryptogenic stroke is key. Many aspects of diagnosis and determination of treatment suitability require input from both specialties. The exchange of neurological imaging and cardiological assessment is essential to help avoid inaccurate diagnosis of PFO as a culprit for cryptogenic stroke, and skills from both neurological and cardiological settings are valuable in selecting patients who are most likely to benefit from intervention. In routine practice, it is considered that diagnosis might be most effectively based on ESUS criteria. In patients with proven DVT and risk of stroke, a combination of anticoagulation and PFO closure requires consideration. 
Sharing of best practice and familiarity with available treatment options is also pivotal in selecting the most beneficial strategy for a patient. Patients managed primarily in a neurology setting may have reduced access to PFO closure than those seen in conjunction with an interventional cardiologist. It has also been observed that a younger patient managed in the neurology setting may not be screened for VTE in the acute phase of stroke, which is of clear importance to patients with a PFO and cryptogenic stroke; a lack of insight into the presence of VTE may also contribute to suboptimal medical therapy selection, if the patient is managed using antiplatelet agents rather than anticoagulants. A multidisciplinary team may also consider the potential benefit of PFO closure for older ESUS patients in whom atherosclerosis is excluded by medical history and ongoing assessment. Similarly, the benefits on migraine burden in patients with a PFO might also be taken into account in the management strategy.

Through coordination of multidisciplinary care teams, management of cryptogenic stroke can move away from an observational and non-interventional approach and towards a more timely and effective strategy. PFO closure represents a once-in-a-lifetime intervention protecting against the recurrence of certain types of stroke. ${ }^{55}$ Greater awareness of the technique may play a role in reducing ischemic stroke recurrence and thus the burden of repeated strokes throughout the patient's remaining lifetime.

\section{Concluding remarks}

The understanding of cryptogenic stroke and ESUS, and the capacity for their diagnosis is improving constantly. With the support of an effective multidisciplinary team, the early determination of culprit versus incidental PFO will help inform early and effective management strategies. Personalization of medicine is key in this complicated field; however, the emerging evidence base for the use of PFO closure, particularly in younger patients at risk of recurrent cryptogenic stroke, provides support for this intervention. Challenges remain in the selection of patients for PFO closure in routine practice, especially for older patients or patients with a history of lacunar stroke. We welcome the ongoing evolution of treatment guidelines and await further long-term evidence to develop our understanding of the benefits of PFO closure and the selection of peri- and post-intervention medical therapy, to help inform optimized management strategies and improve outcomes in patients at risk of recurrent stroke. $\square$
1. Li L, Yiin GS, Geraghty OC, et al. Incidence, outcome, risk factors, and long-term prognosis of cryptogenic transient ischaemic attack and ischaemic stroke: a population-based study. Lancet Neurol. 2015:14:903-13.

2. Hart RG, Diener HC, Coutts $\mathrm{SB}$, et al. Embolic strokes of undetermined source: the case for a new clinical construct. Lancet Neurol. 2014;13:429-38.

3. Kottoor SJ, Arora RR. Cryptogenic stroke: to close a patent foramen ovale or not to close? I Cent Nerv Syst Dis. 2018;10:1179573518819476.

4. Amarenco P, Bogousslavsky J, Caplan LR, et al. New approach to stroke subtyping: the A-S-C-O (phenotypic) classification of stroke Cerebrovasc Dis. 2009:27:502-8.

5. Nouh A, Hussain M, Mehta T, Yaghi S. Embolic strokes of unknown source and cryptogenic stroke: implications in clinical practice. Front Neurol. 2016;7:37.

6. Amarenco P, Bogousslavsky J, Caplan LR, et al. The ASCOD phenotyping of ischemic stroke (updated ASCO phenotyping). Cerebrovasc Dis. 2013:36:1-5.

7. Yaghi S, Bernstein RA, Passman R, et al. Cryptogenic stroke: research and practice circ Res. 2017:120:527-40.

8. Ntaios G, Papavasileiou V, Milionis H, et al. Embolic strokes of undetermined source in the Athens stroke registry: a descriptive analysis. Stroke. 2015;46:176-81.

9. Homma S, Sacco RL. Patent foramen ovale and stroke. Circulation 2005;112:1063-72.

10. Belkin RN, Kisslo J. Atrial septal aneurysm: recognition and clinical relevance. Am Heart J. 1990;120:948-57.

11. Saver $\mathrm{JL}$, Mattle HP, Thaler D. Patent foramen ovale closure versus medical therapy for cryptogenic ischemic stroke: a topical review. Stroke. 2018:49:1541-8.

12. Anantha-Narayanan M, Anugula D, Das G. Patent foramen ovale closure reduces recurrent stroke risk in cryptogenic stroke: a systematic review and meta-analysis of randomized controlled trials. World J Cardiol. 2018;10:41-8.

13. Overell JR, Bone I, Lees KR. Interatrial septal abnormalities and stroke: a meta-analysis of case-control studies. Neurology. 2000;55:1172-9.

14. Diener HC, Gerloff C, Thaler DE, Wohrle J. Closure of patent foramen ovale and cryptogenic stroke: unresolved issues. Curr Neurol Neurosci Rep. 2018;18:92

15. Alsheikh-Ali AA, Thaler DE, Kent DM. Patent foramen ovale in cryptogenic stroke: incidental or pathogenic? Stroke. 2009;40:2349-55

16. Meier B, Frank B, Wahl A, Diener HC. Secondary stroke prevention: patent foramen ovale, aortic plaque, and carotid stenosis. Eur Heart J. 2012;33:705-13.

17. Saremi F, Emmanuel N, Wu PF, et al. Paradoxical embolism: role of imaging in diagnosis and treatment planning. Radiographics. 2014;34:1571-92

18. Meacham RR, Headley AS, Bronze MS, et al. Impending paradoxical embolism. Arch Intern Med. 1998:158:438-48.

19. Rigatelli $\mathrm{G}$, Rigatelli $\mathrm{A}$. Closing patent foramen ovale in cryptogenic stroke: the underscored importance of other interatrial shunt variants. World I Cardiol. 2015:7:326-30

20. Mojadidi MK, Zaman MO, Elgendy IY, et al. Cryptogenic stroke and patent foramen ovale. J Am Coll Cardiol. 2018;71:1035-43.
21. Katsanos AH, Spence JD, Bogiatzi C, et al. Recurrent stroke and patent foramen ovale: a systematic review and meta-analysis. Stroke. 2014:45:3352-9.

22. Arauz A, Murillo L, Marquez JM, et al. Long-term risk of recurrent stroke in young cryptogenic stroke patients with and without patent foramen ovale. Int J Stroke. 2012;7:631-4.

23. Mas IL, Arquizan C, Lamy C, et al. Recurrent cerebrovascular events associated with patent foramen ovale, atrial septal aneurysm, or both. N Engl J Med. 2001;345:1740-6.

24. Di Tullio MR, Jin Z, Russo C, et al. Patent foramen ovale, subclinical cerebrovascular disease, and ischemic stroke in a population-based cohort. J Am Coll Cardiol. 2013:62:35-41.

25. Lattanzi S, Brigo F, Cagnetti C, et al. Patent foramen ovale and cryptogenic stroke or transient ischemic attack: to close or not to close? A systematic review and meta-analysis. Cerebrovasc Dis. 2018;45:193-203

26. De Rosa S, Sievert H, Sabatino J, et al. Percutaneous closure versus medical treatment in stroke patients with patent foramen ovale: a systematic review and meta-analysis. Ann Intern Med. 2018;168:343-50

27. Mariucci E, Donti A, Salomone L, et al. Recurrent Stroke after transcatheter PFO closure in cryptogenic stroke or TIA: Iong-term follow-up. Cardiol Res Pract. 2017;2017:9849425.

28. Saver JL. Clinical Practice. Cryptogenic stroke. N Eng/ J Med. 2016;374:2065-74

29. Collado FMS, Poulin MF, Murphy JJ, et al. Patent foramen ovale closure for stroke prevention and other disorders. J Am Heart AssoC. 2018:7:e007146.

30. Kuijpers T, Spencer FA, Siemieniuk RAC, et al. Patent foramen ovale closure, antiplatelet therapy or anticoagulation therapy alone for management of cryptogenic stroke? A clinical practice guideline. BMJ. 2018;362:k2515

31. Morais LA, Sousa L, Fiarresga A, et al. RoPE score as a predictor of recurrent ischemic events after percutaneous patent foramen ovale closure. Int Heart J. 2018;59:1327-32.

32. Kent DM, Ruthazer $\mathrm{R}$, Weimar $\mathrm{C}$, et al. An index to identify stroke-related vs incidental patent foramen ovale in cryptogenic stroke. Neurology. 2013:81:619-25.

33. Kernan WN, Ovbiagele B, Black HR, et al. Guidelines for the prevention of stroke in patients with stroke and transient ischemic attack: a guideline for healthcare professionals from the American Heart Association/American Stroke Association. Stroke. 2014;45:2160-236

34. Shariat A, Yaghoubi E, Farazdaghi M, et al. Comparison of medica treatments in cryptogenic stroke patients with patent foramen ovale: a randomized clinical trial. I Res Med Sci. 2013:18:94-8.

35. Messe SR, Gronseth G, Kent DM, et al. Practice advisory: recurrent stroke with patent foramen ovale (update of practice parameter): report of the Guideline Development, Dissemination and Implementation Subcommittee of the American Academy of Neurology. Neurology. 2016;87:815-21.

36. Diener HC, Sacco RL, Easton JD, et al. Dabigatran for prevention of stroke after embolic stroke of undetermined source. N Eng/ J Med. 2019:380:1906-17.

37. Hart RG, Sharma M, Mundl $\mathrm{H}$, et al. Rivaroxaban for stroke prevention after embolic stroke of undetermined source. N Eng/ J Med. 2018;378:2191-201.
38. Diener H, Grau A, Baldus S. Cryptogenic stroke and patent foramen ovale (abridged and translated version). Neurol Res Pract. 2019;1. DOl: https://doi.org/10.1186/s42466-019-0008-2 [Online ahead of print].

39. Furlan AJ, Reisman M, Massaro J, et al. Closure or medical therapy for cryptogenic stroke with patent foramen ovale. N Eng/ I Med. 2012;366:991-9.

40. Meier B, Kalesan B, Mattle HP, et al. Percutaneous closure of patent foramen ovale in cryptogenic embolism. N Engl J Med. 2013;368:1083-91

41. Carroll JD, Saver JL, Thaler DE, et al. Closure of patent foramen ovale versus medical therapy after cryptogenic stroke. N Eng I I Med 2013:368:1092-100.

42. Saver JL, Carroll JD, Thaler DE, et al. Long-term outcomes of patent foramen ovale closure or medical therapy after stroke N Eng/ J Med. 2017;377:1022-32.

43. Mas JL, Derumeaux G, Guillon B, et al. Patent foramen ovale closure or anticoagulation vs. antiplatelets after stroke. N Eng/ J Med. 2017;377:1011-21.

44. Sondergaard L, Kasner SE, Rhodes JF, et al. Patent foramen ovale closure or antiplatelet therapy for cryptogenic stroke N Engl J Med. 2017;377:1033-42.

45. Lee PH, Song JK, Kim JS, et al. Cryptogenic stroke and high-risk patent foramen ovale: the DEFENSE-PFO trial. J Am Coll Cardio. 2018;71:2335-42.

46. Shah R, Nayyar M, Jovin IS, et al. Device closure versus medica therapy alone for patent foramen ovale in patients with cryptogenic stroke: a systematic review and meta-analysis. Ann Intern Med. 2018:168:335-42.

47. Pristipino C, Sievert $H$, D'Ascenzo F, et al. European position paper on the management of patients with patent foramen ovale. General approach and left circulation thromboembolism Eurolntervention. 2019;14:1389-402.

48. Wein $\mathrm{T}$, Lindsay MP, Cote R, et al. Canadian stroke best practice recommendations: secondary prevention of stroke, sixth edition practice guidelines, update 2017. Int I Stroke. 2018;13:420-43.

49. Witt DM, Nieuwlaat R, Clark NP, et al. American Society of Hematology 2018 guidelines for management of venous thromboembolism: optimal management of anticoagulation therapy. Blood Adv. 2018;2:3257-91.

50. Meier B, Nietlispach F. The evil of the patent foramen ovale: we are seeing but the tip of the iceberg. Eur Heart J. 2018:39:1650-2.

51. Zaman MO, Mojaddedi S, Nietlispach F, et al. PFO-mediated stroke: exposing the misnomer of "cryptogenic" stroke. Am J Cardiol. 2019;123:2059-60

52. Diener HC, Eikelboom J, Connolly SJ, et al. Apixaban versus aspirin in patients with atrial fibrillation and previous stroke or transient ischaemic attack: a predefined subgroup analysis from AVERROES, a randomised trial. Lancet Neurol. 2012;11:225-31.

53. Meier B. Closure of patent foramen ovale: technique, pitfalls, complications, and follow up. Heart. 2005;91:444-8.

54. Merkler A, Gialdini G, Yaghi S, et al. Long-term risk of complications after percutaneous transcatheter closure of patent foramen ovale (S51.003). Neurology. 2017;88:S51.003.

55. Nietlispach F, Meier B. Percutaneous closure of patent foramen ovale: an underutilized prevention? Eur Heart J. 2016;37:2023-8. 